Article

\title{
Why Do(n't) People Move When They Get Older? Estimating the Willingness to Relocate in Diverse Ageing Cities
}

\author{
Hannah C. Haacke ${ }^{1, *}$, Friederike Enßle ${ }^{1}$, Dagmar Haase ${ }^{1}$, Ilse Helbrecht ${ }^{1}$ and Tobia Lakes ${ }^{1,2}$ \\ ${ }^{1}$ Department of Geography, Humboldt-Universität zu Berlin, 12489 Berlin, Germany; \\ E-Mails: hannah.haacke@geo.hu-berlin.de (H.C.H), friederike.enssle@hu-berlin.de (F.E.), dagmar.haase@geo.hu-berlin.de \\ (D.H.), ilse.helbrecht@geo.hu-berlin.de (I.H.), tobia.lakes@geo.hu-berlin.de (T.L.) \\ 2 IRI THESys, Humboldt-Universität zu Berlin, 10099 Berlin, Germany \\ * Corresponding author
}

Submitted: 14 December 2018 | Accepted: 10 April 2019 | Published: 18 June 2019

\begin{abstract}
Two of the dominant processes shaping today's European cities are the ageing and diversification of the population. Given that the range of action usually decreases in later life, the living environment around the place of residence plays an important role in the social integration of the older generation. Hence, spatial patterns of residence indicate the extent of opportunities for the older population to engage in urban life and, therefore, need to be addressed by urban planning and policy. The aim of this article is to study the interrelation between diversity in later life-in terms of migrant history, gender, social class, and age-as well as planned and actual (past) movements of elders. We have chosen Berlin as a case study and draw from a quantitative survey with elders (age $60+$ ) from diverse backgrounds $(N=427)$. Our results from descriptive analysis and statistical hypothesis tests show that age impacts people's past and planned movement; we observe a peak in the decisions to move at the age of 65-75 and a drop in the inclination to move among people over 80 . None of the other factors is similarly influential, but we observe appreciable tendencies regarding the impact of gender and social class on planned movements. Our study suggests that variables other than classic socio-demographic data, such as apartment size, rent, social networks, and health, and their interrelations may offer a promising starting point for achieving a full picture of older people's movement behaviour.
\end{abstract}

\section{Keywords}

ageing cities; Berlin; diversity; elders; moving behaviour; survey; urban planning

\section{Issue}

This article is part of the issue "The City, Aging and Urban Planning", edited by Matthias Drilling (University of Applied Sciences and the Arts Northwestern Switzerland) and Fabian Neuhaus (University of Calgary, Canada).

(C) 2019 by the authors; licensee Cogitatio (Lisbon, Portugal). This article is licensed under a Creative Commons Attribution 4.0 International License (CC BY).

\section{Introduction}

Demographic change and diversification of the population are two developments that alter the social patterns of European cities. Increased life expectancy, social disparities, and transnational flows of migration lead to great diversity among older people in terms of age, social class, ethnicity, migrant background, and gender (Calasanti, 1996; Pain, Mowl, \& Talbot, 2000). This poses new questions for planning and governance on the cre- ation of age-friendly cities that respond to the needs of older people with different backgrounds.

With age, people are likely to become frailer and thus increasingly dependent on their neighbourhood (Cramm, van Dijk, \& Nieboer, 2018). When experiencing mobility loss, access to facilities in proximity to home gets more important (Menec, Means, Keating, Parkhurst, \& Eales, 2011), because unless social infrastructures, public transport, and health care are in the vicinity, elders may not be able to access them at all. In response to 
decreasing action range in later life, meaning that elders spent most of their time at home or close to their homes (Baltes, Maas, Wilms, Borchelt, \& Little, 1999), urban politicians and planners need to know where older people live and whether they are planning to relocate. Only then can planning and governance ensure proximity to health care and social services. Earlier studies focusing on ageing in place suggest that older people tend to stay in familiar surroundings as long as possible and feel especially attached to their home and neighbourhood (Rowles, Oswald, \& Hunter, 2003; Scharf, Phillipson, \& Smith, 2005). Nevertheless, research on relocation at old age indicates an increase in the number of elders who change their place of residence (Kricheldorff, 2017; Zimmerli, 2016). Reasons for relocation in later life are manifold and comprise environmental, socioeconomic, health-related, social, psychological, space, and time dimensions (Roy, Dubé, Després, Freitas, \& Légaré, 2018). Early theories on relocation at old age distinguish between voluntary and involuntary moves, such as Wiseman's (1980) behavioural model that names forced movements due to decreasing functional abilities, financial status, and need for care. There is also Litwak and Longino's (1987) "Migration Patterns" that refers to the amenities move when people move shortly after retiring in order to improve their lifestyle and gain access to friends. Later studies refer to voluntary and involuntary moves as push and pull factors (Perry, Shen, \& Gonzales, 2018). They report poor health, isolation, insufficient support, and feelings of insecurity as pushing factors, while factors that pull elders to relocate are, for example, attractive locations and the longing to be near friends and a certain community (Bekhet, Zauszniewski, \& Nakhla, 2009). Smetcoren et al. (2017) analyse how socio-demographic and socio-economic factors, as well as kinship and health, impact both push and pull factors. They conclude that elders with lower household income and poor mental health are more affected by pushing factors while elders with higher income and homeowners are more likely to relocate due to pulling factors such as an attractive environment. Their findings suggest that the analysis of social diversity helps to understand who moves in later life and why.

Other studies deepen the knowledge on sociodemographic factors and relocation: Social class, in terms of education and income, influences the relocation of elders. While a lower income hinders movement in later life (Hayward, 2004; Sommers \& Rowell, 1992; Teti, Kuhlmey, Dräger, \& Blüher, 2012; Zimmerli, 2016), a higher level of education fosters the willingness to move (Biggar, 1980; Teti et al., 2012; Zimmerli, 2016). According to literature, the impact of age differs according to planned movement and actual movement. While studies on the willingness to relocate found that plans to relocate decrease with increasing age (Hansen \& Gottschalk, 2006; Teti et al., 2012; Zimmerli, 2016), studies on accomplished movement do not show the same tendencies (Hansen \& Gottschalk, 2006; Sommers \& Rowell,
1992). Earlier studies have demonstrated that gender clearly affects the willingness to relocate (Choi, 1996; Krout, Holmes, \& Erickson, 2003; Sommers \& Rowell, 1992; Teti et al., 2012). All studies have found that women are more willing to move than men in later life. Perry et al. (2018) analyse the impact of ethnicity on relocation at an old age, suggesting that low education and home-ownership reduces the likelihood to relocate among older black adults while older white adults refrain from relocation in later life if they are in poor health condition or own a house and have a strong social network in their neighbourhood. Besides the aforementioned socio-demographic factors, earlier experiences in moving, as well as engagement in activities and social life, foster plans to move, while high housing satisfaction and withdrawal from social engagement hinder relocation (Zimmerli, 2016).

Larger survey studies such as the German Ageing Survey (DEAS, 2014) or a survey by the city of Berlin that analyses the quality of life, interests, and independence in later life (LISA), include no questions on planned or accomplished movements in later life (Bezirksamt Mitte von Berlin, 2010). Available statistical data in Berlin provides information on the movement of the population regarding migrant background, gender, and age. However, there are no additional diversity variables, such as ethnic diversity, migration channel, or variables concerning social class (StatIS, 2017). Thus far, few studies have deeply engaged with the diversity of older people in Western Cities (see Calasanti, 1996; Enßle \& Helbrecht, 2018). Therefore, we lack knowledge about the effects of a society getting older and, simultaneously, more diverse. It is against this backdrop that our study seeks to analyse the interrelations between willingness to move in later life and diversity in terms of gender, age, social class, and migrant background. Our study adds to the existing knowledge as we analyse planned and past movement in later life across different countries of birth, nationalities, migration channels, age, levels of education, and income. By examining these interrelating factors, we seek to better understand how diversity affects decisions to relocate at old age. More specifically, we aim to answer the following questions: To what extent do age, social class, and migrant history influence actual and planned movements? Further, what reasons might cause older people from different backgrounds to move?

\section{Research Design and Methods}

\subsection{Case Study}

We chose Berlin as a case study for this research. Berlin is the capital of Germany and has approximately 3.7 million inhabitants. Among those are 900,000 inhabitants aged 60 years or older (StatIS, 2017). These elders are the target group in this case study. We chose Berlin because it is a big, dynamic city with many older people from heterogeneous and diverse backgrounds (see Table 1). 
The historical division of Berlin leads to diversity among older migrants, comprising former guest workers from Mediterranean areas (former West-Berlin) and Socialist countries such as Vietnam, Angola, and Cuba (former East-Berlin). The project builds on two empirical steps: (1) qualitative, hypothesising research, and (2) a quantitative, hypothesis-testing survey to derive basic principles for an agent-based model that would allow for exploring future ageing cities.

\subsection{Questionnaire and Sample}

To address the research questions, we mainly relied on a survey on diversity and ageing that we conducted with elders in Berlin in 2018, because existing datasets do not provide enough information, neither on moving behaviour nor on social diversity. In addition, we included findings from 18 expert interviews that we conducted with representatives from different counselling centres, social initiatives, and social and cultural meeting places in 2017. We used expert interviews to gain first insights into the nexus between ageing and diversity to guide the following research. We interviewed experts from social initiatives and cultural centres for e.g., Turkish, Arabic, Russian, Vietnamese, Polish Elders; public counselling centres for elders with low income and three housing projects for respective older gays and lesbians as well as older females. The interviews followed an exploratory, open approach and comprised questions on housing conditions, challenges of the ageing process, social networks, and the influence of gender, ethnicity, religion, sexuality, (dis-)ability, and social class on the ageing experience. We analysed the interviews according to the qualitative content analysis after Mayring (2000). To compare the expert's perspective with the everyday life experience of older people, we discussed the main findings from the interviews in four focus groups with 26 elders in total.

The qualitative findings helped us develop our hypotheses for the quantitative survey and to adjust the research approach to our target group: people aged 60 years and above from different social and ethnic backgrounds. We chose 60 years as the age limit to include the change from working life to retirement (Engstler \& Romeu Gordo, 2017). As ethnic minorities tend to be underrepresented in quantitative surveys (Feskens, Hox, Lensvelt-Mulders, \& Schmeets, 2006), we refrained from a classic household survey. Rather, we used contacts that we had established earlier in our qualitative research as starting points and distributed the questionnaires through a snowball system. We asked our interview partners to distribute the questionnaire among their clients and included further institutions and groups that they recommended.

Figure 1 shows the distribution of the elders who answered the questionnaire in the 447 planning units of Berlin. A planning unit is smaller than a ZIP area. There is no cluster of answers in areas where the questionnaires had been distributed originally (distribution centres). Surveys were returned from areas in the city centre as well as from the suburban areas and cover former eastern and western parts of Berlin.

To raise the response rate among older migrants, we provided the questionnaire in eight languages: German, English, Turkish, Arabic, Polish, Russian, Bosnian, and Vietnamese. We chose these because the city of Berlin's nurs-

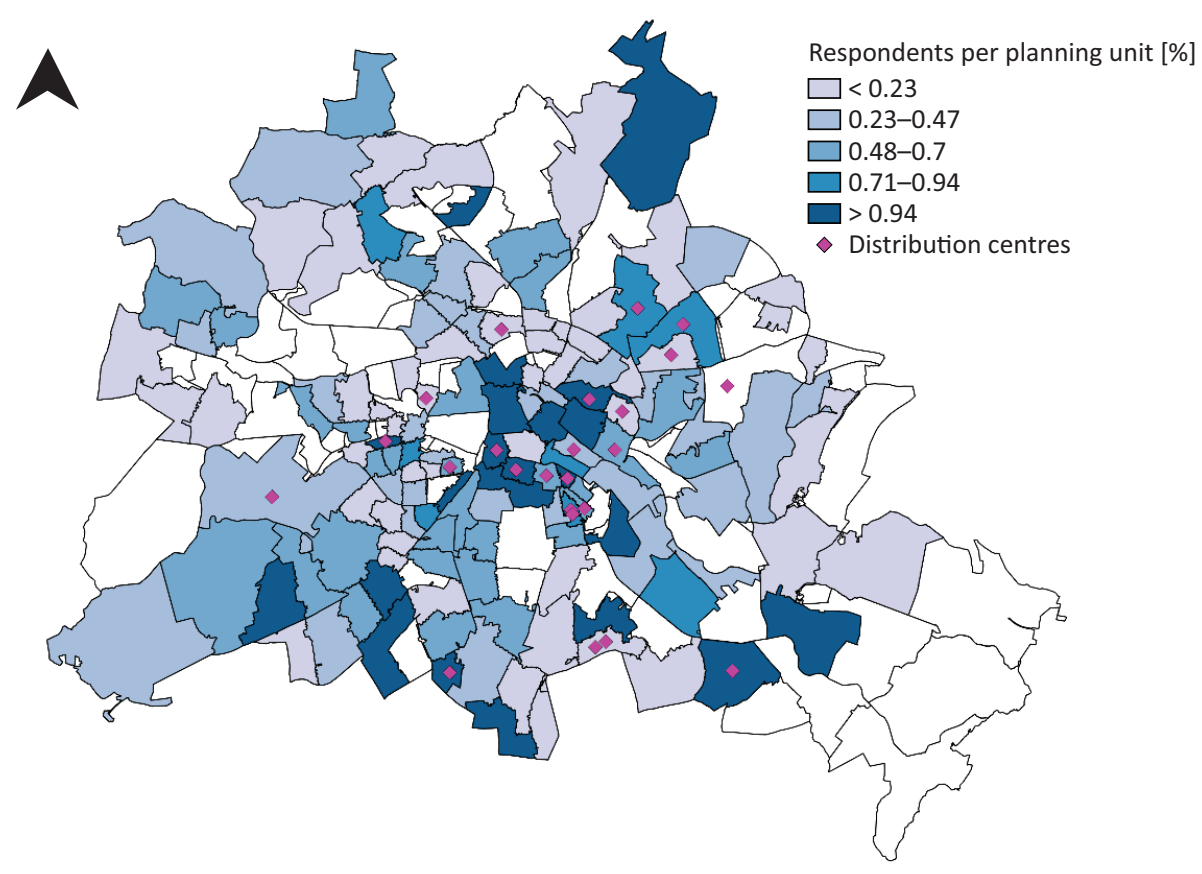

Figure 1. Distribution of respondents proportionate to all elders who answered the questionnaire and distribution centres in Berlin (Basemap: StatIS, 2017). 
ing support centres publishes their information in these languages; therefore, it is likely that the majority of elders (or one of their relatives) speaks one of those. Accredited translators provided the translation. Even though every translation implies interpretation, we ensured comparable content of the questionnaires through pre-tests and careful checking with bilingual elders. We distributed our questionnaire in paper format and as an online questionnaire via the distribution centres.

Our questionnaire comprised four thematic sections in total-(1) older people and society, (2) social environment in later life, (3) changes with the end of work life, and (4) housing in old age-and a section on sociodemographic data. For this article, we analysed data from section (4) and the socio-demographic data (see appendix). To estimate if older people plan to move in the future, we asked: 'Do you sometimes think about moving somewhere else?' If this was answered positively, the following question was asked: 'For what reasons do you want to move?' For the answers, we offered ten reasons, as well as 'other' (see Section 3). The questionnaire also asked about the most recent movement and the reasons for it. If this most recent movement had happened since the person turned 60, it was included in the analysis as a past movement. As our analysis includes both, plans to move in the future and past (accomplished) movements since a respondent turned 60 , the term 'willingness to move' refers to accomplished as well as panned movements. We do not differentiate whether the motivation to move was voluntary or forced.

We distributed 786 questionnaires in paper format and links to the online version via six organisations (a mailing list for older Gays and Lesbians, a mailing list of Berlin seniors' delegation, a centre for intercultural care in later life, a computer club, a mailing list of a housing project, and a mailing list of Berlin's community management institutions). Afterwards, we received 668 responses (475 online and 193 in paper format). This resulted in a response rate of $24.5 \%$ for the paper format. The exact response rate of the online version is unknown due to privacy issues pertaining to the organisation's mailing lists. After the exclusion of missing data and respondents younger than 60 years, our sample includes 427 participants. Of the completed questionnaires, 143 were completed on paper and 284 were answered online. Our sample comprised 279 female and 148 male participants, 374 participants who were born in Germany and 45 participants who were not born in Germany. In the following, we define people with migrant background as those who were not born in Germany, regardless of their nationality. A total of 395 questionnaires were completed in German and 32 in one of the languages mentioned above. The majority of respondents belonged to the $65-75$ age group (32\% were $65-70 ; 24 \%$ were $70-75$ ), $15 \%$ were $60-65$ years old, and about $8 \%$ were over 80 ( $7 \%$ were $80-85,1 \%$ were $85-90$, and $0.7 \%$ were above 90; see Table 1 ).

Table 1 shows a comparison of the sample from our questionnaire with population data from the Federal Statistical Office of Berlin (ER) for those aged 60 and older (StatIS, 2017). Note that the Federal Statistical Office differentiates people with migrant background and Immigrants (nationality other than German and/or at least one parent without a German nationality (StatIS, 2017). Our research does not distinguish between the two groups and our definition of migrant background only includes

Table 1. Characteristics of the survey population (in \%).

\begin{tabular}{|c|c|c|c|}
\hline Categories & & Survey (2018) & ER population (2018) \\
\hline \multirow[t]{2}{*}{ Migratory Status } & German & 87.1 & 86 \\
\hline & Migrant Background & 11.5 & 14 \\
\hline \multirow[t]{2}{*}{ Gender } & Female & 65.3 & 55.7 \\
\hline & Male & 34.7 & 44.4 \\
\hline \multirow[t]{6}{*}{ Marital Status } & Married & 42.2 & 54.3 \\
\hline & Divorced & 18.5 & 16.1 \\
\hline & Widowed & 16.9 & 20 \\
\hline & Single & 12.6 & 9.2 \\
\hline & Civil Union & 1.6 & 0.4 \\
\hline & Other & 7.5 & $<0.1$ \\
\hline \multirow[t]{7}{*}{ Age } & $60-64$ & 15.2 & 22.2 \\
\hline & $65-69$ & 32.1 & 20.5 \\
\hline & $70-74$ & 23.7 & 17.1 \\
\hline & $75-79$ & 20.4 & 19.2 \\
\hline & $80-84$ & 6.6 & 12.2 \\
\hline & $85-89$ & 1.2 & 5.7 \\
\hline & Above 90 & 0.7 & 3.2 \\
\hline \multirow{3}{*}{ Education } & Low education & 4.2 & - \\
\hline & Medium education & 32.3 & - \\
\hline & High education & 46.8 & - \\
\hline
\end{tabular}


elders who were born in another country, therefore the number of People with migrant Background of the ER population sums up the number of immigrants and people with a migrant background. That means that an entire comparison of the two datasets is not possible. In comparison to former studies conducted in Berlin (see, e.g., DEAS, 2014), our sample adequately represents the older population of Berlin, particularly older people with and without a migrant background. More females than males answered the questionnaire. However, there is an underrepresentation of people over 80 years.

\subsection{Hypotheses}

We drew on the findings of our qualitative study from 2017 and literature to derive the following hypotheses on the influence of age, social class, migrant history, and gender on people's past and planned movement.

Age: In accordance with earlier studies and our qualitative data, we assumed that with increasing age, the willingness to relocate would decrease (Kemper, 2001; Teti, Grittner, Kuhlmey, \& Blüher, 2014; Zimmerli, 2016). Furthermore, we presumed a peak of decisions to move at the age of $65-70$ because people usually enter retirement at that age.

\subsubsection{Social Class}

Drawing on literature and our qualitative findings, we derived the hypothesis that a small income hinders movement (Hayward, 2004; Teti et al., 2012; Zimmerli, 2016), while a high level of education fosters willingness to move (see Biggar, 1980; Hayward, 2004; Sommers \& Rowell, 1992; Zimmerli, 2016). We defined social class by household income (very low income: $<800 €$, very high income: >5000€) and education level in line with the International Standard Classification of Education (ISCED; UNESCO Institute for Statistics, 2012).

\subsubsection{Migrant History}

We frame 'migrant history' from three angles: migrant background (country of birth other than Germany), nationality, as well as channel of migration (e.g., former guest worker, refugees, former students, etc.). Since to our knowledge, no research exists on migrant history and relocation in later life, we based our hypothesis on our qualitative research. It suggests that the channel of migration determines life chances and social inclusion in the host society, so it is likely to determine the ability to move, too. We presume that migrant background and nationality have no effect on the elder's relocation.

\subsubsection{Gender}

Earlier studies point to connections between gender and willingness to move (see, e.g., Teti et al., 2012), but we could not find a plausible connection between gender and willingness to relocate in later life in our qualitative research. To explore the contradiction between the state of the art and our findings, we included gender in our analysis.

As earlier studies argue that elders move to escape isolation and loneliness (Bekhet et al., 2009), we included family status (married; divorced; in partnership; widowed; in same-sex partnership) in our analysis to test whether it has any influence on the moving behaviour.

\subsection{Data Analysis}

We applied descriptive statistics to explore the survey data. We began by identifying three groups: older people who are planning to move (category 'planned movement'); older people who have already moved since they turned 60 ('past movement'); and older people who wish to neither move nor have moved before they turned 60 . To test our hypothesis, we consecutively analysed our data in terms of social class, migrant history, and age. Descriptive analysis and statistical tests are used to test our hypothesis for planned movements and for past movements. It is unclear if elders who plan to move will really move. Therefore, we test the dependency between elders who belong to the category 'past movement' and 'planned movement' and the recommendation to a friend to move into the area. Our hypothesis comprises two cases: (1) that elders would not recommend friends to move into their area if they themselves want to move elsewhere, and (2) that they would recommend friends to move into their area if they recently moved there or do not want to move anymore.

\subsection{Statistics}

We chose three different tests for our mixed dataset for testing dependencies between variables: $\chi^{2}$-test, analysis of variance (ANOVA) and multivariate binomial logistic regression.

Firstly, $\chi^{2}$-tests were used to test the relationship between two categorical variables. Usually, the null hypothesis $\mathrm{H}_{0}$ is that the variables are independent while $\mathrm{H}_{1}$ means that variables are dependent (Kabacoff, 2015). The $p$-value is the measure of dependency, and if $p<0.05$, the relationship is significant with a probability of $95 \%$ (James, Witten, Hastie, \& Tibshirani, 2013).

Secondly, ANOVA has been used to test the relationship between metric and categorical variables with the F-Test (Dormann, 2013). We did a one-way ANOVA because there is only one classification variable (Kabacoff, 2015).

Thirdly, multivariate binomial logistic regression has been used to test the non-linear influence of several variables on an independent variable. Contrary to linear regression, categorical and binary parameters can be tested and non-linear functions are allowed as predictors (Kabacoff, 2015). The statistical analysis was conducted with $\mathrm{R}$ in R-Studio. 


\section{Results}

From our descriptive data analysis, we know that $46 \%$ of all elders from our survey (200 from 427) plan to move or have already moved. Note that these two events are not mutually exclusive, i.e., some people have already moved but plan to move again. Among all elders, there are $26 \%$ who plan to move and $26 \%$ who have already moved.

Figure 2 shows the age distribution of elders for past and planned movement. For past movements, the analysis shows that there is a peek at the age of 67; then, the number of movers remains comparatively high, has another peak at 70, and drops afterwards. There are only a few elders who have moved after they turned 80 . Note that for past movement we considered the age of the participants at the time of movement. Therefore, it is possible that in some cases someone moved at a certain age even though no one who participated in the survey is of this age. This can be seen in Figure 2 for the age of 89 . There are also peaks of planned movement at $65,70,71$, and 73 . After reaching age 80 , none of our respondents is planning to move.

We cannot find any clear association between past or planned movement and migrant background: $47.1 \%$ of all people without a migrant background and $46.7 \%$ of those with a migrant background are willing to move. In some cases, older people from a certain country have a higher willingness to move, but we only get a tendency because of the small number of cases $(N=45)$ when the dataset is split into the different countries. The only group that wants to move more often consists of those who have left their country because of bad living conditions $(55 \%, N=11)$. We obtained 43 responses stating the reasons for migration. German language skills and length of stay in Germany did not show any impact on the willingness to move.

From our descriptive analysis, we find that $48.4 \%$ of all females and $43.9 \%$ of all males plan to move. Household income leads to less willingness to move when the monthly income is very low $(<800 €)$ or very high $(>5000 €)$. People who have an income between $800 €$ and $5000 €$ per month have more or less the same willingness to move. By contrast, there is a lower willingness to move the lower the education is. People with a high education want to move in $51.5 \%$ of cases, people with a medium education want to move in $44.9 \%$ of all cases, and people with a low education want to move in $38.9 \%$ of cases.

We found some tendencies for family status. Elders who are single $(61 \%, N=54)$, divorced $(53 \%, N=79)$, or in a homosexual relationship ( $80 \%, N=10)$ have a higher moving willingness than elders who live in another relationship. However, only a few people who are living in a homosexual relationship answered the questionnaire.

The results of our statistical hypothesis tests are listed in Tables 2, 3, and 4. In Table 2, we demonstrate that there is a dependency between age and planned and past movement. In Table 3, the results of the $\chi^{2}$-test are listed. There are dependencies between planned

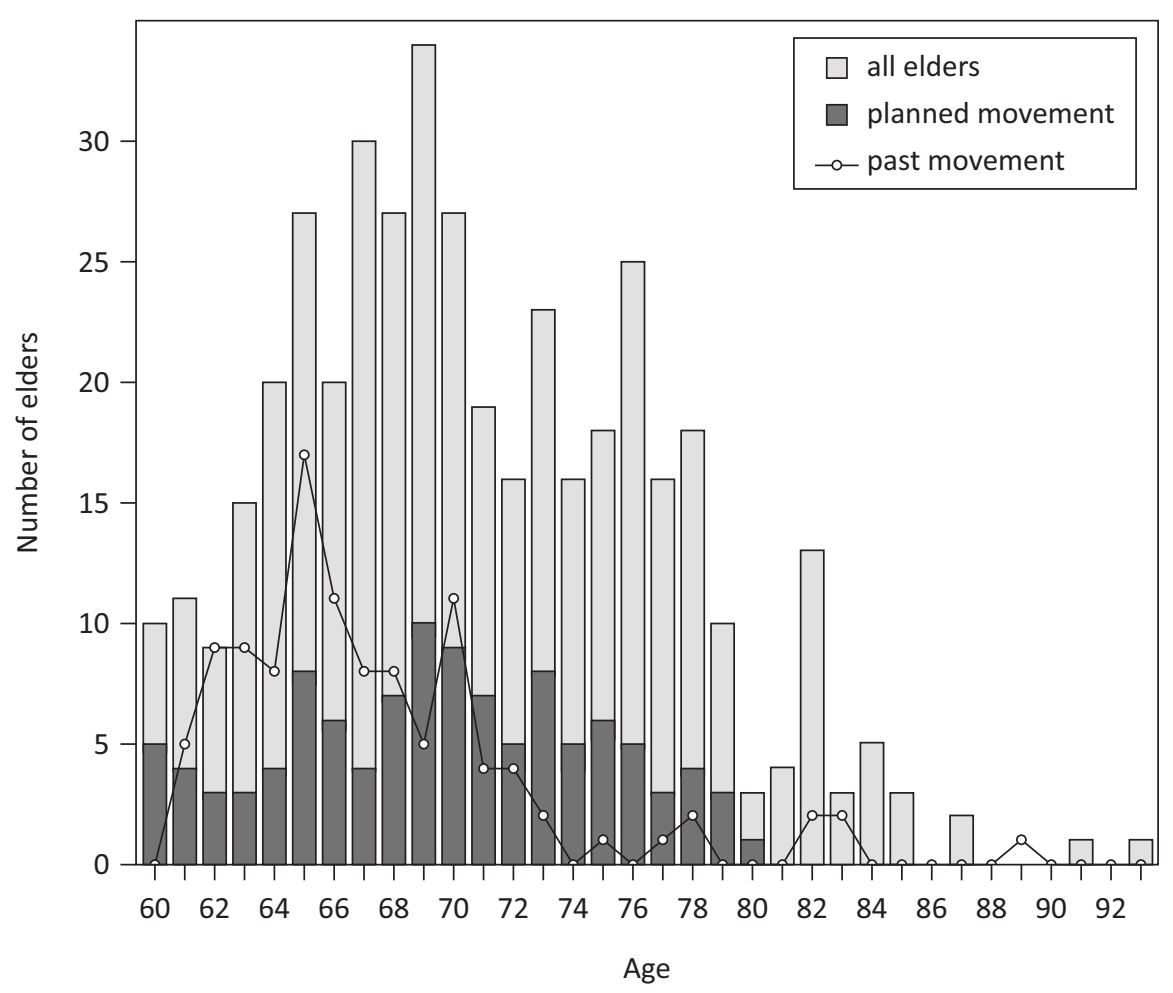

Figure 2. Total age distribution of the respondents, the age distribution of people who plan a movement, and distribution of moving age for past movement. 
Table 2. Results of the analysis of variance (ANOVA).

\begin{tabular}{lll}
\hline Dependent variable & Independent variable & P-Value \\
\hline Age & Planned movement & $*$ \\
Age & Past movement & $* * *$ \\
\hline
\end{tabular}

Note: $\mathrm{p}<0.05^{*}, \mathrm{p}<0.01^{* *}, \mathrm{p}<0.001^{* * *}$.

Table 3. Results of the $\chi^{2}$-test.

\begin{tabular}{lll}
\hline Dependent variable & Independent variable & P-Value \\
\hline Planned movement & Gender & $*$ \\
Past movement & Gender & Migrant background \\
Planned movement & Migrant background & $* * *$ \\
Past movement & Recommendation for friends to move into the area & $* *$ \\
Planned movement & Recommendation for friends to move into the area & $* *$ \\
Past movement & Family status & $* *$ \\
Planned movement & Family status & \\
Past movement & ISCED & \\
Household income & &
\end{tabular}

Note: $\mathrm{p}<0.1^{*}, \mathrm{p}<0.05^{* *}, \mathrm{p}<0.005^{* * *}$.

Table 4. Results of the multivariate binomial logistic regression with interaction between household income and ISCED.

\begin{tabular}{llllll}
\hline Dependent variable & Predictor I & Reference group & Log odds & Std. error & P-Value \\
\hline Planned movement & ISCED: low & ISCED: medium; moving: yes & 1.6864 & 1.608 \\
Planned movement & ISCED: high & ISCED: medium; moving: yes & 1.1415 & 0.5285 & $*$ \\
Planned movement & Household income & ISCED: medium; moving: yes & 0.0004 & 0.0002 &. \\
Past movement & ISCED: low & ISCED: medium; moving: yes & 3.0306 & 2.0704 \\
Past movement & ISCED: high & ISCED: medium; moving: yes & 0.2687 & 0.4995 \\
Past movement & Household income & ISCED: medium; moving: yes & 0.0002 & 0.0002 & \\
\hline
\end{tabular}

Note: $\mathrm{p} \sim 0 * * *, \mathrm{p}<0.001^{* *}, \mathrm{p}<0.01^{*}, \mathrm{p}<0.05$.

movement, gender, family status, and if the elder would recommend friends to move into the area. Dependencies for past movement exist for elders if they would recommend friends to move into the area. There is a dependency between categorised household income and the ISCED as can be seen in Table 3. There are no dependencies between past or planned movement and migrant background.

There are dependencies, measured with multivariate binomial logistic regression (Table 4), between planned movement, high education, and household income. The log odds of the interaction values are low, which is caused by the low interaction values of household income and are, therefore, not included.

\subsection{Reasons for Movement}

Figure 3 lists the most frequently mentioned reasons for relocation. Age-related reasons for movement, such as planning to move into a nursing home or the fear of being unable to care for oneself, were rarely mentioned. More important are apartment-related factors such as apartment size, rent, or lack of handicapped access. If past and planned movements are summarised, then movement because the apartment is not obstacle-free is one of the most important reasons for movement (past $18.7 \%$, planned 21\%). Moving to a smaller apartment is the leading reason for past movement $(25.6 \%)$. Other reasons for past movement are other reasons (5.5\%), moving to assisted living (4.6\%), moving into a shared accommodation (4.1\%), and movement to Berlin (2.3\%). Reasons for planned movement are bad connection with the train (4.4\%), annoying living environment (3.9\%), other reasons (3.9\%), change of the living situation (3.9\%), flat is too small $(2.2 \%)$, no longer being able to live alone (2.2\%), and movement to assisted living (1.1\%).

\section{Discussion}

Our results show that almost half of the older people in our survey plan to move or have already moved. This contrasts with earlier studies, which stress the reluctance of older people to change their place of residence (Kemper, 2001; Scharf et al., 2005), but resonates with findings on the willingness of elders to relocate with the start of retirement (Kricheldorff, 2017; Litwak \& Longino, 1987; Zimmerli, 2016). Even if we consider only respondents who put their intention into practice and actually moved after turning 60 , a quarter of our sample still moved. The difference in our findings may be related to the destina- 


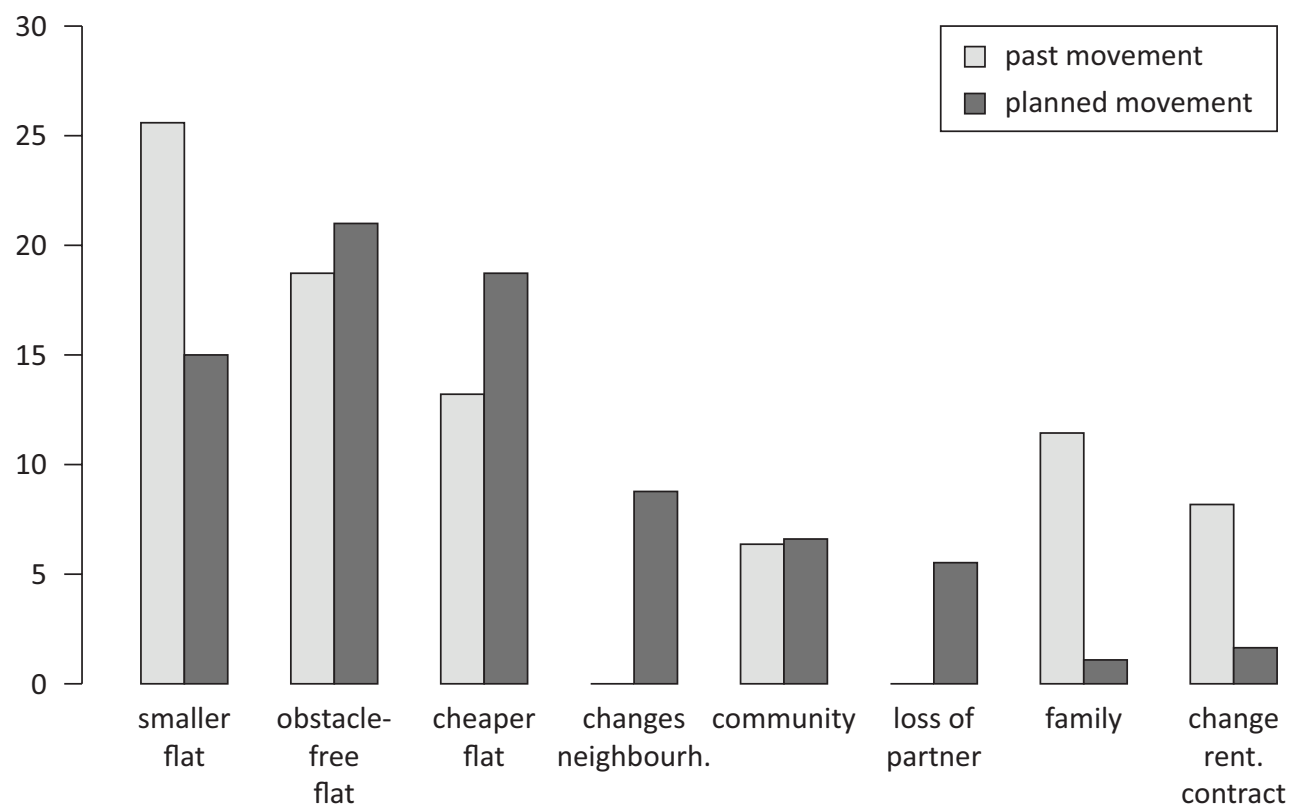

Figure 3. Main reasons for movement (\% of respective movement type (past or planned), multiple answers possible).

tion of movement. While earlier studies tend to focus on movements to nursing homes and assisted living apartments (e.g., Nay, 1995; Taylor, Osterman, Will Acuff, \& $\emptyset$ stbye, 2005; Teti et al., 2012), our respondents moved (or planned to move) into apartments with lower rent or that were smaller or closer to their social networks. We cannot exclude that the elders moved into an old age residential home, but the main reason for movement is that the new flat is cheaper. In addition, the tense housing market in Berlin is likely to affect the comparatively high proportion of elders who consider moving. Both the consideration to move and actual movement might result from gentrification and fear of being displaced rather than from a voluntary decision.

\subsection{Do Age, Social Class, and Migrant History Influence the actual and planned movements?}

Our results on the association between diversity-age, social class, and migrant history-and willingness to relocate reveal patterns for age. Results from the ANOVA analysis show that there is a dependency between age and moving behaviour, which resonates with other studies (Hansen \& Gottschalk, 2006; Teti et al., 2012). Our descriptive analysis supports our hypotheses that, with increasing age, willingness to relocate decreases and that there is a peak in the decision to move at $65-70$ years. This links to the start of the official retirement age of 67 and fits Litwak and Longino's amenities move (1987). Age affects the inclination to move, but only until people turn 80. From a descriptive analysis, we know that people who are older than 80 do not plan to move at all. This finding confirms earlier studies that show high residential stability among the very old (Rowles et al., 2003). Therefore, we conclude that there is an influence of age on moving behaviour across people with different backgrounds.
In addition, our qualitative research indicates that social class-understood as level of education and income-influences decision making and behaviour in later life as income impacts the ability and intention to move in terms of the possibility or pressure to move, depending on income and rising rents. Furthermore, the household income influences plans for movement. When we combine education with household income, we observed some dependencies between social class and movement behaviour: There is a dependency between the plan to move and high education with according household income, but no dependency between past movement and social class. The result that elders with high education and income plan to move more often overlaps with other research (Hayward, 2004; Sommers \& Rowell, 1992).

With regard to migrant history, we presumed based on our qualitative research that channel of migration would likely determine willingness to move, whereas a person's country of migrant background would not have an effect. In our descriptive analysis, we did not find any dependencies between migrant background/no migrant background and willingness to move. That was confirmed by the $\chi^{2}$-test, as there is no dependency between the two variables. In our descriptive analysis, we found some evidence for the assumption that country of birth and nationality affect willingness to relocate. We also determined that the channel of migration had no impact on an older person's inclination to relocate. However, the numbers of respondents with specific countries of birth, nationalities, or migrant channels is low (e.g., eight people were born in Turkey and six were born in Bosnia), even though the proportion of elders with migrant background who answered the questionnaire nearly matches the proportions from the ER $(11.5 \%$ in survey to $14 \%$ in ER). This means that no reliable state- 
ment can be made based on the detailed breakdown of the different countries of origin or the migrant channel.

In the descriptive analysis, the gender of our respondents gives only a tendency of possible movement, showing that older females tend to be more willing to move than are older males. However, in the $\chi^{2}$-test we found a dependency between planned movement and gender, meaning that gender has an influence on planning behaviour but not on movements that actually happened. This contradicts our initial assumption that there is no plausible connection between gender and willingness to relocate that we draw from our qualitative research, as the statistical analysis reveals the influence of gender on willingness to relocate. Our findings partly overlap with earlier studies (Krout et al., 2003) that confirm that gender influences the moving behaviour of older people. However, our finding that gender influences future movements contradicts Hansen and Gottschalk (2006), who find no connection between thoughts of moving and gender. Further studies should more deeply explore the differing impact of gender on planned movements as opposed to accomplished movements and related reasons. Apart from that, we found other variables that have an influence on moving behaviour, such as family status. Our findings suggest that elders that are living alone (divorced or separated) have a higher willingness to move. In the future, these aspects need to be analysed in more detail, for example, why a certain family status leads to certain moving behaviour. However, we did not find any dependencies between past movement and other variables. The reason for that may be that decisions are more complex and cannot be described with one or two variables, and factors that are more complex and their interrelation have to be taken into account.

\subsection{Why Do the Elders Move?}

Our research shows that desire for a smaller apartment, an obstacle-free apartment, and the need to move to a cheaper apartment are the top three reasons for movement (see Figure 3). Although elders with different backgrounds participated in our survey, all three reasons can be explained by ageing rather than by diversity: The desire to move to an obstacle-free apartment in later life is quite plausible because, Germany-wide, less than $3 \%$ of apartments are equipped for people with reduced mobility (Nowossadeck \& Engstler, 2017). The large number of elders who move into smaller flats is a bit surprising because a movement into a smaller flat usually leads to higher housing costs due to increasing rents. Nevertheless, this seems not to be an issue in our sample. One possible explanation is the large number of elders with good education and possible higher income, which enables movement. In addition, some older people intend to move because of increasing rents. This could be a Berlin-specific result, given the tense situation of Berlin's housing market and on-going gentrification (see Holm, 2013), but since the average rent in Germany for people aged $40-85$ increased between 1996 and 2006 by $57 \%$ (Nowossadeck \& Engstler, 2017), the problem of rising rents also affects people across the country and possibly even abroad.

As income, usually decreases in retirement, older people are particularly vulnerable when it comes to gentrification and rental increases. These developments could lead to more movement among older people when they are forced to move into cheaper apartments or it could reduce movement because an old rental contract guarantees a relatively low rent. Consequently, moving to a smaller apartment could mean moving to an apartment with higher rent. That might explain that more people plan to move because of increasing rents than actually moved. Elders are not able to find a cheaper flat somewhere else and stay in their current apartment. This also helps to explain the low number of people with low income intending to relocate. It is quite plausible that older people with an income lower than $800 €$ per month cannot find any affordable apartments to move to. Our data also reveals older people's desire to age close to other older people, be it in special housing projects or in a neighbourhood of one's friends. This finding is in accordance with other studies pointing to the growing importance of social networks and friendship in later life (Böger, Huxhold, \& Wolff, 2017).

To predict and evaluate the movement behaviour of elders, it might be helpful to differentiate between voluntary and involuntary factors because such an approach could point to possible destinations and reasons for movement (see Perry et al., 2018; Wiseman, 1980). Both aspects are partially covered in our survey, yet hard to differentiate.

Given that their prolonged lifespan now means that 'the elders' comprise an age group spanning nearly four decades, it becomes increasingly important to take motivations for movement other than age-related factors into account. Furthermore, it may be useful to split elders into smaller age cohorts, such as 'young old' $(<80)$ and 'old old' (>80).

\subsection{Limitations}

The findings of our study are limited by the fact that people with low education are underrepresented. For a thorough statistical analysis, the sample size of people with a migrant background is too small to account for the different specific countries of birth and nationalities among the elders in Berlin. Thus, our hypothesis concerning migrant backgrounds cannot be answered conclusively despite our sample nearly representing the actual percentage of elders with migrant background in the population. In addition, the method of distributing the survey in counselling centres and meeting places for elders is likely to address a well-connected community and probably explains the underrepresentation of people aged $80+$. It is also possible that the respondents misunderstood the question about household income and stated their indi- 
vidual and not the combined income, which complicates any direct comparison.

Furthermore, it is difficult to estimate if elders who said that they sometimes consider moving will actually move. We tried to add reliability by comparing the dependency of planned movement with the question of whether elders would recommend friends to move into the area. Our findings show that there is a dependency between recommendation and planned movement, and so it is likely that a recommendation/no recommendation might lead to a stay in the area/movement to another area. However, there are still many factors that will influence future movements. Therefore, other aspects might be considered in the future as well (e.g., how close doctors are, or if there are parks close by), to make a prognosis on planned movement even more reliable.

Another limitation for the calculation of dependencies is that there are only a few respondents if the sample is split into smaller groups. Affected by this is, apart from the country of origin, low education $(N=24)$, people who live in a homosexual relationship $(N=10)$, and people who live in a relationship without being married $(N=17)$. The larger the number of respondents per category, the more reliable the estimated probabilities will be. The small number of respondents in the low education category, therefore, can be the reason for the high $p$-values in Tables 3 and 4 . If the number of respondents is low, the resulting calculated $p$-values might not reflect the true $p$-values of the hypothesis and a significant relationship might exist in some of the cases (Casella \& Berger, 2002).

A further aspect is that we were not able to cover all influencing factors in our survey to limit its overall length. We selected factors based on literature review and expert interviews, which we think are the most interesting and influential concerning diversity and mobility. However, to get a complete picture, further studies are needed to cover other aspects, such as the need for care or decreasing mobility in later life.

In this article, we did not analyse where the elders are moving to, as the focus lies on the current location and why an older person might want to move. The next step would be to analyse what the preferred destinations are, and whether certain groups have different targets than others. When this step is concluded, a prognosis of the development of the spatial pattern is possible.

\section{Conclusions}

The inhabitants of European cities are becoming both older and more diverse. As the everyday life of older people primarily takes place around their place of residence (Baltes et al., 1999), the key for age-friendly communities lies in the immediate living environment. Urban politicians and planners need to know older people's plans to relocate in order to ensure health care and social services nearby. Therefore, the aim of this article was to estimate the extent to which diversity in terms of age, gen- der, social class, and migrant history affects older people's willingness to relocate. Drawing on a quantitative survey from Berlin with 427 respondents, our analysis shows that age is one of the variables that affect willingness to move. We observed a peak in movements in the 65-70 age group and a drop in willingness to relocate at the age of 80 . Small tendencies are visible with regard to gender in the descriptive analysis, as females show a slightly higher willingness to move. However, gender only has an influence on planned movement and not on actual movements according to dependency tests.

In addition, testing social class and its influence on movement shows that elders with high education plan to move more often, which also overlaps with findings of others (Teti et al., 2012; Zimmerli, 2016). We did not find any dependency between low education and willingness to move. A potential future research direction would be an analysis of leading factors of differences in planned and past movements, including research on voluntary and involuntary moves. Usually, elders with higher education have higher income and, therefore, more possibilities to move. However, the effort might be too high, which leads to no movement in the end because they might be able to cope with more push-factors due to their high income.

Reflecting on our initial objective of estimating how diversity in later life-in terms of age, social class, migrant history, and gender-affects willingness to relocate, we conclude that age clearly affects willingness to relocate, which could indicate a particular importance of age ahead of other differences. One might argue that the ageing process affects everyone equally, especially when it comes to very old age. Physical and mental constraints come to the fore and people experience similar change and meet similar challenges, regardless of their social and cultural background. In addition, it seems necessary to analyse age groups separately and not 'elders' as a single group. Other factors, such as gender and education, need to be analysed in detail in future studies since they show some tendencies concerning willingness to move.

We conclude that the 'classical' variables we usedsocial class, gender, age, and migrant history-are not sufficient to make general statements about the movement behaviour of older people. Other factors and their interrelations need to be included, as already conducted when using the variable 'social class' and its influence on past and planned movement. Considering the reasons our respondents gave for their motivation to move, such as moving to apartments that are accessible to the handicapped, smaller, or cheaper, it might be more appropriate to form groups based on people's physical condition, their social networks, or the size of their apartments.

Future research should start here and explore the impact of these less common variables on the willingness to relocate. It should also engage deeply with the interrelations between well-known variables. To identify influential variables, it will be helpful to analyse motivations be- 
hind the willingness to move and rethink categories that are quite naturally used to group people. Given the complexity of the variable 'migrant history' for example, we recommend the application of a qualitative methodology to understand connections between migration-related experiences and willingness to relocate in later life. To enable planning and city administrations to respond appropriately to the existing willingness to move among older people, more research should address motivations for movement as well as destinations of relocation. As the broad age group of 'the elders' encompasses nearly four decades, not all movements are into nursing homes. Alternative destinations, such as projects for convivial ageing, small and easily accessible apartments, or quiet and green neighbourhoods, deserve more attention in research and practice. Knowing the determining factors behind older people's willingness to relocate, their motivations, and their preferred destinations is a first step to creating cities and communities that respect manifold needs and wishes of people in later life and providing liveable neighbourhoods for all generations.

\section{Acknowledgments}

Funding was provided by the German Research Foundation (DFG, Project number HE 2417/16-1 and HA 3484/ $8-1$ ). The funding source had no involvement in any stage of the research. Many thanks to Lisa Johanna Thiele and Carlotta Marie Reh for distributing and collecting the questionnaires and to all participants in the project. We would like to thank three anonymous reviewers for improving this article with their contributions.

\section{Conflict of Interests}

The authors declare that there is no conflict of interest.

\section{References}

Baltes, M. M., Maas, I., Wilms, H. U., Borchelt, M., \& Little, T. D. (1999). Everyday competence in old and very old age: Theoretical considerations and empirical findings. The Berlin aging study: Aging from, 70. In P. B. Baltes \& K. U. Mayer (Eds.), The Berlin aging study: Aging from 70 to 100 (pp. 384-402) New York, NY: Cambridge University Press.

Bekhet, A. K., Zauszniewski, J. A., \& Nakhla, W. E. (2009). Reasons for relocation to retirement communities: A qualitative study. Western Journal of Nursing Research, 31(4), 462-479.

Bezirksamt Mitte von Berlin. (2010). Lebensqualität, Interessen und Selbstständigkeit im Alter (LISA): Ergebnisse einer Befragung älterer Menschen im Bezirk Mitte von Berlin [Quality of life, interests and independence in age: Results of a survey with elder people in the disctict Mitte in Berlin]. Beiträge zur Gesundheitsförderung und Gesundheitsberichterstattung, 16, 1-60.
Biggar, J. C. (1980). Who moved among the elderly, 1965 to 1970. Res Aging, 2, 73-91.

Böger, A., Huxhold, O., \& Wolff, J. K. (2017). Wahlverwandtschaften: Sind Freundschaften für die soziale Integration wichtiger geworden? [Elective affinities: Is friendship becoming more important for social integration?] In K. Mahne, J. K. Wolff, J. Simonson, \& C. Tesch-Römer (Eds.), Altern im WandelZwei Jahrzehnte Deutscher Alterssurvey (DEAS) [Aging through the course of time: Two decades German Ageing Survey] (pp. 257-271). Wiesbaden: Springer.

Calasanti, T. M. (1996). Incorporating diversity: Meaning, levels of research, and implications for theory. The Gerontologist, 36(2), 147-156.

Casella, G., \& Berger, R. L. (2002). Statistical interference. Belmont, CA: Brooks/Cole Cengage Learning.

Choi, N. G. (1996). Older persons who move: Reasons and health consequences. Journal of Applied Gerontology, 15, 325-344. https://doi.org/10.1177/ 073346489601500304

Cramm, J. M., van Dijk, H. M., \& Nieboer, A. P. (2018). The creation of age-friendly environments is especially important to frail older people. Ageing \& Society, 38(4), 700-720.

DEAS. (2014). Scientific use file deutscher alterssurvey (SUF DEAS) 2014, Version 1.0. Deutsches Zentrum für Altersfragen. Retrieved from https://doi.org/10. 5156/DEAS.2014.M.001

Dormann, C. (2013). Parametrische Statistik: Verteilungen, maximum likelihood und GLM in $R$ [Parametric statistics: Distributions, maximum likelihood and GLM in R]. Freiburg: Springer Spektrum.

Engstler, H., \& Romeu Gordo, L. (2017). Der Übergang in den Ruhestand: Alter, Pfade und Ausstiegspläne [The transition into retirement: Age, paths and exit plans]. In K. Mahne, J. K. Wolff, J. Simonson, \& C. TeschRömer (Eds.), Altern im Wandel-Zwei Jahrzehnte Deutscher Alterssurvey (DEAS) [Aging through the course of time: Two decades German Ageing Survey] (pp. 65-80). Wiesbaden: Springer.

Enßle, F., \& Helbrecht, I. (2018). Ungleichheit, Intersektionalität und Alter(n)-Für eine räumliche Methodologie in der Ungleichheitsforschung [Inequality, intersectionality and age(ing): For a spatial methodology in research on inequality]. Geographica Helvetica, 73, 227-239.

Feskens, R., Hox, J., Lensvelt-Mulders, G., \& Schmeets, H. (2006). Collecting data among ethnic minorities in an international perspective. Field Methods, 18(3), 284-304.

Hansen, E. B., \& Gottschalk, G. (2006). What makes older people consider moving house and what makes them move? Housing, Theory and Society, 23(1), 24-54.

Hayward, L. M. (2004). Mid-life patterns and the residential mobility of older men. Canadian Journal on Aging, 23, 73-89.

Holm, A. (2013). Berlin's gentrification mainstream. In M. Bernt, B. Grell, \& A. Holm (Eds.), The Berlin reader. 
A compendium of urban change and activism (pp. 171-188). Berlin: transcript.

James, G., Witten, D., Hastie, T., \& Tibshirani, R. (2013). An introduction to statistical learning with applications in R. New York, NY: Springer science + business media.

Kabacoff, R. I. (2015). $R$ in action: Data analysis and graphics in $R$. Shelter Island, NY: Manning.

Kemper, F.-J. (2001). Wohnformen, Altersstruktur, Lebenszyklusphasen [Living arrangements, age structure and life cycle]. Berichte zur Deutschen Landeskunde, 75(2/3), 137-146.

Kricheldorff, C. (2017). Strukturelle Rahmenbedingungen für neue Wohn-und Pflegearrangements im Alter [Structural framework conditions for new living and nursing arrangements in old age]. Zeitschrift für Gerontologie und Geriatrie, 50(3), 185-186.

Krout, J. A., Holmes, H., \& Erickson, M. A. (2003). Residential relocation. In J. A. Krout, \& E. Wethington (Eds.), Residential choices and experiences of older adults: Pathways to life quality (pp. 27-48). Berlin: Springer.

Litwak, E., \& Longino, C. F. Jr. (1987). Migration patterns among the elderly: A development perspective. Gerontologist, 27(3), 266-272.

Mayring, P. (2000). Qualitative Inhaltsanalyse. Grundlagen und Techniken (7th edition) [Qualitative content analysis. Fundamentals and techniques]. Weinheim: Deutscher Studien Verlag.

Menec, V. H., Means, R., Keating, N., Parkhurst, G., \& Eales, J. (2011). Conceptualizing age-friendly communities. Canadian Journal on Aging/La revue canadienne du vieillissement, 30(3), 479-493.

Nay, R. (1995). Nursing home residents' perceptions of relocation. Journal of Clinical Nursing , 4(5), 319-325.

Nowossadeck, S., \& Engstler, H. (2017). Wohnung und Wohnkosten im Alter [Accomodation and cost of housing in later life]. In K. Mahne, J. K. Wolff, J. Simonson, \& C. Tesch-Römer (Eds.), Altern im WandelZwei Jahrzehnte Deutscher Alterssurvey (DEAS) [Aging through the course of time: Two decades German Ageing Survey] (pp. 287-300). Wiesbaden: Springer.

Pain, R., Mowl, G., \& Talbot, C. (2000). Difference and the negotiation of 'old age'. Environment and Planning D: Society and Space, 18(3), 377-393.

Perry, T. E., Shen, H.-W., \& Gonzales, E. (2018). Further investigations of the social and material convoy: Exploring relationships between race, volunteering, and relocation. Social Work Research, 42(4), 290-300.

Rowles, G. D., Oswald, F., \& Hunter, E. G. (2003). Inte- rior living environments in old age. Annual Review of Gerontology and Geriatrics, 23(1), 167-194.

Roy, N., Dubé, R., Després, C., Freitas, A., \& Légaré, F. (2018). Choosing between staying at home or moving: A systematic review of factors influencing housing decisions among frail older adults. PloS One, 13(1).

Scharf, T., Phillipson, C., \& Smith, A. E. (2005). Social exclusion of older people in deprived urban communities of England. European Journal of Ageing, 2(2), 76-87.

Smetcoren, A.-S., de Donder, L., Dury, S., de Witte, N., Kardol, T., \& Verté, D. (2017). Refining the push and pull framework: Identifying inequalities in residential relocation among older adults. Ageing \& Society, 37(1), 90-112.

Sommers, D., \& Rowell, K. (1992). Factors differentiating elderly residential movers and nonmovers: A longitudinal analysis. Population Research and Policy Review, 11, 249-262.

StatIS. (2017). Einwohnermelderegister [Resident's registration office]. Das Statistische Informationssystem Berlin-Brandenburg. Retrieved from https:// www.statistik-berlin-brandenburg.de/Statistiken/ statistik_met.asp?Ptyp $=650 \&$ Sageb $=12041 \& \mathrm{creg}=$ BBB\&anzwer $=10$

Taylor, Jr., Osterman, D. H., Will Acuff, J., S., \& Østbye, T. (2005). Do seniors understand their risk of moving to a nursing home? Health Services Research, 40(3), 811-828.

Teti, A., Grittner, U., Kuhlmey, A., \& Blüher, S. (2014). Wohnmobilität im Alter [Residential mobility in old age]. Zeitschrift für Gerontologie und Geriatrie, 47(4), 320-328.

Teti, A., Kuhlmey, A., Dräger, D., \& Blüher, S. (2012). Prädiktoren individueller Wohnmobilität älterer Frauen und Männer [Predictors of individual residential mobility in older women and men]. Prävention und Gesundheitsförderung, 7(4), 278-285.

UNESCO Institute for Statistics. (2012). International standard classification of education ISCED 2011. Montreal: UNESCO Institute for Statistics.

Wiseman, R. F. (1980). Why older people move: Theoretical issues. Research on Aging, 2, 141-154.

Zimmerli, J. (2016). Wohnmobilität im Alter-ein zukunftsfähiger Ansatz für die Siedlungsentwicklung nach innen? [Residential mobility of the elderly: A sustainable approach to higher residential density?] disPThe Planning Review, 52(3), 61-72.

\section{About the Authors}

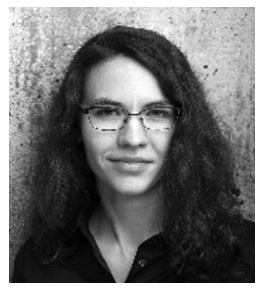

Hannah C. Haacke is a PhD candidate and Research Associate in the Applied Geoinformation Science lab at the Geography Department of Humboldt-Universität zu Berlin. She received the BSc degree in Geographical Sciences from the Freie Universität Berlin in 2013 and the MSc degree in Geodesy and Geoinformation Science, with a major in Geoinformation Science from the Technische Universität Berlin in 2016. She is currently working on models that describe the development of the spatial distribution of population groups. 


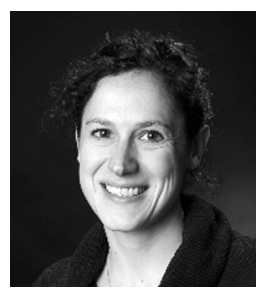

Friederike Enßle is a PhD Student and Research Associate in the Cultural and Social Geography lab at the Geography Department of Humboldt-University in Berlin. She holds a bachelor's degree in Area Studies (Central Asian Studies) and a Master's Degree in Urban Geography. Her current research focuses on the interplay of age(ing) and diversity from a spatial perspective.

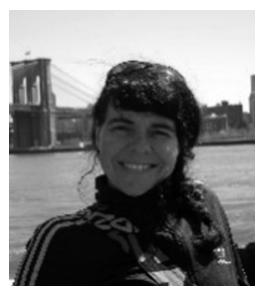

Dagmar Haase is a Professor in Landscape Ecology and Urban Ecology at the Geography Department of Humboldt-Universität zu Berlin, and a Guest Scientist at the Helmholtz Centre for Environmental Research-UFZ, Leipzig, Germany. Her core expertise is in modelling urban land use change, urban system dynamics, and urban telecouplings. Dagmar's focus is also on the quantification and assessment of ecosystem services and landscape functions using statistics and earth observation data.

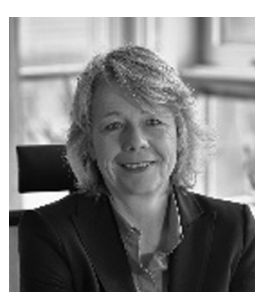

Ilse Helbrecht is a Professor of Cultural and Social Geography at the Geography Department of the Humboldt-Universität zu Berlin since 2009. She received the Caroline von Humboldt Professorship in regard to an Excellence Initiative in 2018. This year, the former director of the Georg-Simmel centre for Metropolis Research was selected as Fellow for the Thomas Mann House of the Federal Republic of Germany in Los Angeles.

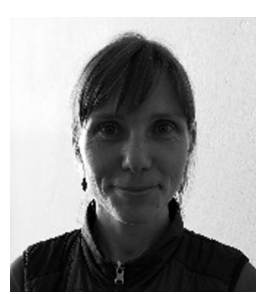

Tobia Lakes is a Professor of Applied Geoinformation Science at the Geography Department of Humboldt-Universität zu Berlin since 2015. Previously, she had an assistant professorship at the Department. Tobia Lakes's expertise is in modelling human-environment interactions in urban areas. She has a strong focus on spatio-temporal modelling techniques combining different data sources. 


\section{Appendix}

\section{Survey 2018}

Block IV: Living in old age

With the next questions, we want to cover your living situation and what you like or dislike about your apartment/house.

13. What year did you live move into your current apartment/house?

14. With whom do you live?

Please tick every box that applies.

$\square \quad$ I live alone.

with my partner

with my child/children

others:

with my grandchild/grandchildren

with my parents

with friends

15. Do you live in a
rental
flat
own property
other:

16. Approximately how much do you pay per month for your apartment/house? Please consider additional cost like electricity, heating, fees for garbage.

17. On which floor do you live? (Ground floor $=0$ )

18. Do you have an elevator in your building?

Yes

No

19. To what extent do the following statements apply to you? Please check:

\begin{tabular}{|c|c|c|c|c|c|}
\hline In my residential area, & Strongly agree & Agree & Partly & Disagree & Strongly disagree \\
\hline ... there are enough stores. & $\square$ & $\square$ & $\square$ & $\square$ & $\square$ \\
\hline ... there are enough doctors and pharmacies. & $\square$ & $\square$ & $\square$ & $\square$ & $\square$ \\
\hline ... I sometimes feel unsafe. & $\square$ & $\square$ & $\square$ & $\square$ & $\square$ \\
\hline ... I am well connected to buses and trains. & $\square$ & $\square$ & $\square$ & $\square$ & $\square$ \\
\hline ... there are enough parks and green areas. & $\square$ & $\square$ & $\square$ & $\square$ & $\square$ \\
\hline
\end{tabular}

20. How often do you use the parks and green areas in your neighbourhood?
(nearly) daily
rarely more than $1 x$ per month
never

$\square \quad 1-3 x$ per week
$\square \quad 1-3 x$ per month

21. Are the parks and green areas accessible and easily reached by foot?
yes
$\square \quad$ with constraints
no 
22. Would you recommend to a good friend to move into your residential area?

yes, because

no, because

undecided

23. When was the last time you moved?

24. What was the postal code of your previous residential area?

If you moved before the postal code in Berlin was changed in 1993, you can also write in the two-digit postal code.

Postal code:

25. What were the reasons for your last move?

Moving into a smaller apartment

Moving into a barrier-free apartment

Moving into a cheaper apartment

Termination of the landlord

Moving into a form of assisted living (e.g. senior citizen housing)

Moving into a shared apartment

Moving to a family member

Other reasons:

26. Do you think sometimes about moving somewhere else?

yes $\rightarrow$ continue with question 27

no $\rightarrow$ continue with question 28

\section{For what reasons do you want to move?}

In my apartments there are steps e.g. thresholds, entrance to shower.

The apartment is too big.

The apartment is too small.

The connection to bus and trains is bad.

The rent is too high.

The neighbourhood changed.

Loss of partner.

I can no longer manage alone.

There is no suitable outpatient care service nearby.

I can move to a care facility.

Other reasons:

\section{V: Personal information}

\section{In which year were you born?}

\section{Are you...}

male

female

different sex

30. What is your current postal code? 
31. Are you...

$\square$ married

divorced

widowed

living separated

in partnership

Others:

32. In which state were you born?

33. What is your nationality?

34. Since when have you been living in Germany?

I was born in Germany. $\rightarrow$ continue with question 36

for years

35. If you were not born or raised in Germany:

I came to Germany...

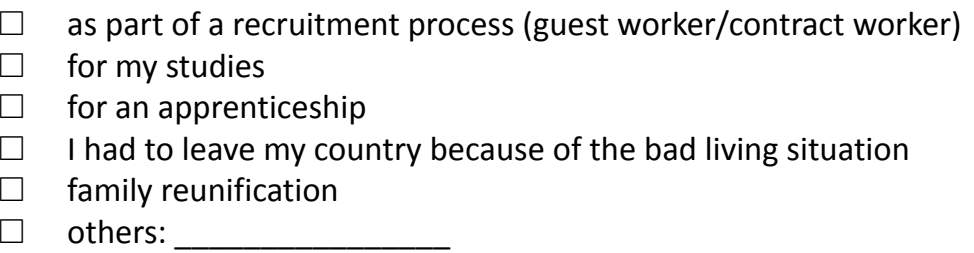

36. How would you rate your knowledge of the German language?

$\square \quad$ German as mother tongue

$\square$ fluent

$\square \quad$ very good to good

I get along

\section{How many years did you attend school?}

38. What is your highest school-leaving qualification?

$$
\text { I do not have one. }
$$

39. What is your highest training qualification (e.g. assistant (Geselle), foremen (Meister) or final degree)? I do not have a training qualification.

40. What profession did you practice last/are you practicing?
$\longrightarrow \quad \square$ homemaker

41. How much is your monthly net household income approximately? 


\section{COGITATIO}

42. How much were you disturbed or impaired in the last seven days by the following problems or discomforts?

\begin{tabular}{|c|c|c|c|c|c|}
\hline & Not at all & A bit & Quite & Strongly & A lot \\
\hline $\begin{array}{l}\text { Physical limitations (e.g. problems while walking or } \\
\text { taking stairs, back pains) }\end{array}$ & $\square$ & $\square$ & $\square$ & $\square$ & $\square$ \\
\hline Diabetes & $\square$ & $\square$ & $\square$ & $\square$ & $\square$ \\
\hline Chronic pains & $\square$ & $\square$ & $\square$ & $\square$ & $\square$ \\
\hline The feeling of being lonely & $\square$ & $\square$ & $\square$ & $\square$ & $\square$ \\
\hline $\begin{array}{l}\text { Insomnia (e.g. problems to find sleep or } \\
\text { sleeping through the night) }\end{array}$ & $\square$ & $\square$ & $\square$ & $\square$ & $\square$ \\
\hline
\end{tabular}

43. How would you describe your state of health?

$\square \quad$ very good

$\square$ good

$\square \quad$ rather bad

$\square$ okay

44. Do you have a care level (Pflegestufe)?

$\square \quad$ Yes $\square \quad$ No

45. Do you have a severely handicapped pass (Schwerbehindertenausweis)?

Yes $\square$ No 